\title{
An indecent proposal or improved quality of bioanalytical data in clinical studies
}

...it is sad that within regulated bioanalysis today, there is little room for experiments and few opportunities to test alternative bioanalytical approaches.

Keywords: bioanalysis $\approx$ clinical $\|$ criteria $\|$ criterion $\approx$ duplicates $\approx$ praxis $\approx$ precision

Since the late 1970s and early 1980s, technology in bioanalysis has developed tremendously. I think the most obvious improvement is the increase in sample analysis throughput. However, my aim in this article is to show that the bioanalytical data obtained in those old days were not qualitatively inferior, but to some extent were superior to the data obtained in contemporary bioanalysis. Approximately 30 years ago, samples in clinical pharmacokinetic (PK) investigations were often analyzed as duplicates, a procedure that has its advantages. This was done despite the low throughput of the available methods. Today's common praxis and guidelines state that PK samples in a clinical study should be analyzed only once and batch-wise, together with calibration samples and quality control (QC) samples. The calibration samples are used for calculating concentrations of the unknowns and the QC samples are used for confirming that the analytical results are relevant. In today's praxis, the quality of the analytical results rely to a great extent on previous validation of the analytical method applied. However, as mentioned above, this has not always been standard procedure and below I discuss and benchmark today's praxis versus how clinical bioanalysis data were obtained in the early days of clinical PK investigations.

\section{Background}

In the late 1970 s and early 1980 s today's praxis would not have been possible due to the lowthroughput of the available methods. It was not unusual that a method had the capacity of only eight to 12 assays per day and required a fulltime employee (FTE). This low capacity did not allow for measurements of calibrators, QC samples and unknowns in the same batch and on the same day. For example, at our laboratory we assayed the calibrators on Monday and the unknowns over the remaining days of the working week, and we had no incorporated QC samples. This meant that we used Monday's calibration graph of 12 data points to calculate the values of the 48 unknowns analyzed over the rest of the week. However, in order to secure the integrity and quality of the PK data, all unknown samples were analyzed at least in duplicate and on different occasions. Praxis was that if the reanalysis diverged more than $10 \%$ from the initial analysis, an additional assay on that sample was performed (triplicate) and the median value was used for PK evaluations. Furthermore, the calibration graphs from several weeks were merged and the merged 'super calibration graph' was not used for calculation until the analysis of the duplicates was initiated. This way of analyzing samples runs the risk that all the duplicates would diverge to a high extent (e.g., due to storage instability of analyte) and that no PK calculation could be performed. However, as this approach was applied in clinical studies, the stabilities of spiked samples in human plasma were well known from preceding preclinical studies and so were the stabilities of incurred samples. In what follows, I propose a hypothetical clinical study in order to discuss a merger of this old-style approach to bioanalysis and our current high-throughput methods.

\section{Proposal}

The proposal is to combine the old duplicate assay concept with today's technology. Let us assume that we were to assay unknown samples with a modern validated high-throughput method without using QC samples and without performing incurred samples reanalysis (ISR) or incurred samples stability (ISS). Instead, we would utilize duplicate assays of the unknowns to obtain an estimate of data reliability and

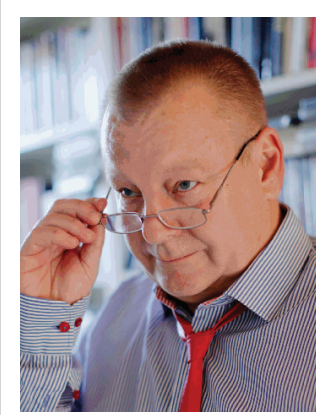

Leif Svensson

Active Biotech AB, Box 724, Scheelevagen 22, SE-220 07, Lund, Sweden

E-mail: leif.svensson@ activebiotech.com 
integrity. This assumption appears as something of a challenge. However, by setting up a purely hypothetical example, I will try to demonstrate how these analytical efforts could be organized in order to achieve more than adequate output in quality and reliability. The example is a study with a total of 1500 samples, a method throughput of 150 unknown samples per day and FTE, the study comprised of 20 batches analyzed over 20 working days.

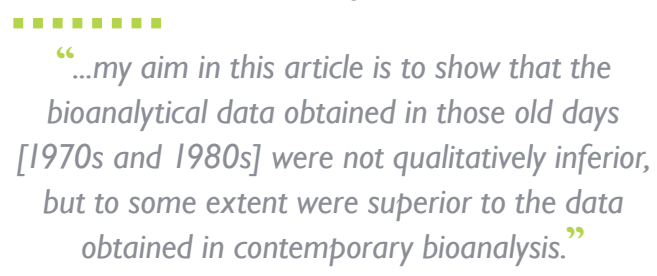

\section{Hypothetical procedure}

The first batch is immediately repeated, such that already in the initial phase of our bioanalytical efforts, we obtain an estimate of whether the precision is within the range that the preceding validation showed. At the same time we will also obtain 150 (10\%) ISR estimates. The following nine batches provide us with the remaining initial estimates and then the remaining nine batches of duplicates are assayed. After 20 batches, the whole dataset can be evaluated and duplicates not within a predefined criterion (e.g., $\pm 15 \%$ of the initial determination) are reassayed as triplicates. The predefined criterion could of course be adjusted to a suitable level, in compliance with study demands, such that a bioequivalence study would require a more stringent criterion than a Phase III study.

\section{Proposed batch criteria}

- If a sample in the duplicate assay diverges more than $\pm 15 \%$ of the initial determination, it is to be assayed once more as a triplicate;

- If more than one-third of determinations in a duplicate batch diverge more than $\pm 15 \%$ of the initial assay, the entire batch is to be assayed once more as a triplicate;

- In a triplicate assay, the median value is reported (not the average, as this will minimize the impact of true outliers in PK calculations) and this third assay must be within $\pm 15 \%$ of one of the preceding assays, otherwise a replicate of four is to be performed;

- When a replicate of four is performed, the relative standard deviation of the three closest estimates must be within $15 \%$, otherwise a sample directed investigation is to be initiated.

\section{Data overview}

The second batch analyzed (i.e., batch one reanalyzed) can almost be considered as a suitability test, because it shows whether data are reproducible or not. If not, the methodology must, of course, be investigated and corrected. The last nine batches give us all the duplicates and an overall estimate of the precision and, in addition, 1350 (90\%) ISS results. If more extended (i.e., prolonged) ISS data are needed, the last batch of duplicates can, of course, be put on hold for a suitable period of time before analysis.

\section{Discussion}

When duplicates are assayed as default, precision in the final data is improved by a factor of 1.4, which must be considered a substantial improvement. The estimate of precision in a validation would rely on six replicates at three levels, repeated on three occasions, which is equal to 54 estimates. However, in the proposed duplicate approach, precision is estimated from 1500 duplicates and this estimate will really reflect the precision of the entire study. Furthermore, the precision estimate is based on real unknowns and not on spiked samples. With today's praxis it could always be questioned whether the precision in the study data is in accordance with the preceding method validation. The correlation of the preceding validation and study data could of course be made more stringent if all study QCs were statistically analyzed, assuming that QC estimates in batches that have been rejected due to improper accuracy are included in the statistics. This is probably often done, however, there is no common criterion upon which the bioanalytical community has agreed.

The proposed duplicate procedure would disclose whether using batch specific calibration graphs or a merged 'super calibration graph' would produce superior data. Merged calibration graphs are more accurate when the variation between graphs is due to random statistical reasons. On the other hand, if there is a true variation between the calibration graphs, one could discuss whether or not the batch variation is propagated to the estimates of the unknowns in the batch. Estimates as duplicates also handle outliers easily without considering fit to PK time concentration curve or other PK properties that require analysis of previous and subsequent 
samples to support a correction or to establish an incomprehensible estimate.

ISR and ISS are produced within the study and ISS will not rely on a small subset of samples but on almost the entire study (ISR is not defined as ISS). This means that it is more likely that ISS tendencies diverging from the initial values will be revealed. Furthermore, if the bioanalysis were to be organized in such a way that the variation of the duplicates may be calculated immediately after analysis, the ISS would be monitored during the study on an almost daily basis, which means that an instability that has turned out to be disclosed and predictable could be compensated for in the end data.

The impact of matrix and matrix components, such as counter ions on sample stability, would be reflected within the study, so if at the beginning of a study one were confident that the method used was unaffected by matrix, the used matrix would not have to be investigated in the initial validation.

However, the above-proposed procedure will require more FTEs in bioanalytical laboratories. If our hypothetical study were to be analyzed according to today's common praxis, approximately $1920(1500+120+150+150)$ estimates would be produced specified as:

- Estimates of unknowns as individuals: 1500 estimates;

- 12 QC samples per batch and ten batches: 120 estimates (at least 5\% of the unknowns, that is 7.5 estimates; however, if we also assume the same number of replicates at all three QC concentration levels, we end up with $4 \times 3=12$ assays);

- 150 ISR, in this example one batch $(10 \%$ of unknowns) is assumed, as truncated batches are inefficient;

- 150 ISS (see motivation above for number of ISR estimates).

This means that the proposed duplicate procedure requires 1080 additional estimates, that is, seven more batches to be assayed. Of course, this implies a substantial cost increase. However, in comparison to the total cost of a clinical study, this additional bioanalytical cost is, in fact, marginal. Also, analysis of duplicates does not increase the workload by a factor of two because much of the work is done prior to the initial analysis: sample identification, organization in batches, sample labeling controls and corrections versus case report forms. Triplicates are not incorporated in the account above, as it is assumed that they are balanced against batches rejected due to QC results not within criterion in today's praxis.

\section{Final remarks}

I gave the title 'An indecent proposal' to this editorial because I recognize that this proposal is unlikely to be neither tested nor validated in comparison to today's praxis. As such, this 'indecent proposal' reflects a traditional scientific rationale that says, 'perform an experiment, then confirm the findings by repeating the experiment and when you succeed, make the findings public for others to discuss and repeat'. However, with its demands for more FTEs and more analytical capacity, the proposal will probably not appeal to managers of bioanalytical laboratories. Besides, clinical studies are very costly and of pivotal importance to the pharmaceutical industry, so there does not seem to be room for explorative activities of this kind. A full-scale test would also require a revision of guidelines if the data should be incorporated in an investigational new drug application. However, from a scientific point of view, it would have been interesting to see if this old procedure from the early 1980 s could match today's praxis and contribute to the quality and integrity of data. In a way, it is sad that within regulated bioanalysis today, there is little room for experiments and few opportunities to test alternative bioanalytical approaches.

Financial \& competing interests disclosure

The author has no relevant affliations or financial involvement with any organization or entity with a financial interest in or financial conflict with the subject matter or materials discussed in the manuscript. This includes employment, consultancies, honoraria, stock ownership or options, expert testimony, grants or patents received or pending, or royalties.

No writing assistance was utilized in the production of this manuscript. 